\title{
DISTRIBUIÇÃO DE POTÁSSIO, MAGNÉSIO E OUTROS METAIS EM MINERAIS DE SEDIMENTOS DO GRUPO BARREIRAS NO MUNICÍPIO DE ARACRUZ, ESTADO DO ESPÍRITO SANTO ${ }^{(1)}$
}

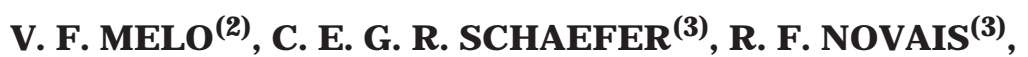 \\ B. SINGH ${ }^{(4)} \&$ M. P. F. FONTES ${ }^{(3)}$
}

\begin{abstract}
RESUMO
Os sedimentos do Grupo Barreiras são muito pobres em minerais primários fontes de nutrientes. Para estudar a distribuição de K, Mg e outros metais em minerais das frações argila, silte e areia, coletaram-se 11 amostras nas profundidades de 0,$7 ; 1,4 ; 2,1 ; 2,8 ; 3,5 ; 4,2 ; 4,9 ; 5,6 ; 7,7 ; 10,5$ e 14,0 m, em um pacote de sedimentos do Grupo Barreiras, no município de Aracruz (ES) (19049'13"S 4016'24"O). Os teores totais de $\mathrm{K}, \mathrm{Mg}, \mathrm{Ca}, \mathrm{Al}, \mathrm{Fe}, \mathrm{Ti}, \mathrm{Mn}, \mathrm{Cu}, \mathrm{Zn}, \mathrm{Ni}, \mathrm{Pb}, \mathrm{Cr}$, $\mathrm{Sr}$ e Ga na terra fina seca em estufa e nas frações argila, silte e areia foram determi nados por espectrômetria de emissão por plasma, após digestão completa da amostra com ácido fluorídrico concentrado. Para avaliar a contribuição de cada espécie mineral nos teores totais de $\mathrm{K}$ e de $\mathrm{Mg}$, amostras da fração argila foram submetidas a extrações seqüenciais e seletivas de minerais. Em virtude da presença de camadas enriquecidas com concreções ferruginosas (princi palmente do tamanho silte), ao longo do pacote de sedi mentos, obtiveramse os maiores teores totais de $\mathrm{Fe}_{2} \mathrm{O}_{3}$, nas profundidades de 2,1; 4,2 e 7,7 m. Os sedimentos do Grupo Barreiras apresentaram limitada reserva de nutrientes, com baixos teores totais de $\mathrm{K}$, Mg e Ca. Verificou-se aumento nos teores de $\mathrm{K}$ na fração argila com a profundidade, atingindo $618,2 \mathrm{mg} \mathrm{kg}^{-1}$ a $14 \mathrm{~m}$. Na fração areia, os teores de $\mathrm{K}$ e de Mg foram maiores nas amostras com maior impureza de quartzo. A reserva em $\mathrm{K}$ e Mg na fração argila foi associada com a presença de mica e caulinita. A contribuição dos óxidos de Fe e de Al, dos aluminossilicatos de baixa cristalinidade e dos óxidos de Fe mais cristalinos, nos teores totais de $\mathrm{K}$ e de Mg, foi inexpressiva. As frações argila e silte foram as principais fontes de metais pesados nas amostras estudadas.
\end{abstract}

Termos de indexação: extração seqüencial, reserva de potássio, reserva de magnésio, metais pesados.

(1) Parte da Tese de Doutorado do primeiro autor. Recebido para publicação em outubro de 2000 e aprovado em maio de 2001.

(2) Professor do Departamento de Solos, Universidade Federal do Paraná - UFPR. Rua dos Funcionários, 1540, J uvevê, CEP 80035-050 Curitiba (PR).

(3) Professor do Departamento de Solos, Universidade Federal de Viçosa - UFV. CEP 36571-000 Viçosa (MG). Bolsista CNPq.

(4) Department of Agricultural Chemistry and Soil Science. The University of Sydney. N.S.W. 2006 Austrália. 


\author{
SUMMARY: DISTRIBUTION OF POTASSIUM, MAGNESIUM AND OTHER \\ METALS IN MINERALS OF BARREIRAS GROUP SEDIMENTS \\ IN THE REGION OF ARACRUZ, ESPÍRITO SANTO STATE, \\ BRAZIL
}

\begin{abstract}
Barreiras Group sediments (Coastal Tablelands) are very poor in primary mineral containing nutrients. To study the distribution of $\mathrm{K}, \mathrm{Mg}$ and other metals in minerals of clay, silt and sand fractions, 11 samples were collected at depths of $0.7 ; 1.4 ; 2.1 ; 2.8 ; 3.5$; $4.2 ; 4.9 ; 5.6 ; 7.7 ; 10.5$ and $14 \mathrm{~m}$, in an exposed sedi mentary sequence of theBarrei ras Group, in Aracruz (19049'13"S 40¹6'24'W), Espírito Santo, Brazil. The total contents of K, Mg, $\mathrm{Ca}, \mathrm{Al}, \mathrm{Fe}, \mathrm{Ti}, \mathrm{Mn}, \mathrm{Cu}, \mathrm{Zn}, \mathrm{Ni}, \mathrm{Pb}, \mathrm{Cr}$, Sr, and $\mathrm{Ga}$ of the bulk sampleand of the day, silt and sand fractions weredetermined by ICP, after a HF extraction. To estimatethecontribution of the mineral species to the total contents of $\mathrm{K}$ and $\mathrm{Mg}$, the clay fraction was submitted to sequential and selectivemineral extractions. Thevariation of thetotal $\mathrm{Fe}_{2} \mathrm{O}_{3}$ with depth in the sediment reveal ed the presence of three plinthic layers, at 2.1, 4.2 and $7.7 \mathrm{~m}$ depth, mainly, in the silt size. Total contents of $\mathrm{K}, \mathrm{Mg}$ and $\mathrm{Ca}$ in the Barreiras Group sediments were very low, indicating a low reserve of these nutrients. Only for $K$, the total nutrient content increased with depth in the clay fraction, reaching $618.2 \mathrm{mg} \mathrm{kg}^{-1}$ at $14 \mathrm{~m}$. The contents of $\mathrm{K}$ and $\mathrm{Mg}$ in thesand fraction weregreater wherelarger quart $\mathrm{z}$ impurity occurred (2.1; 4.2 and $7.7 \mathrm{~m})$. The $\mathrm{K}$ and $\mathrm{Mg}$ reserve in the clay fraction is mainly associated with mica and kaol inite. On theother hand, thecontributi on of Feand Al oxides, aluminosil icate amorphous and crystalline Fe oxides was negligible Clay and silt fractions showed the largest total contents of heavy metals.
\end{abstract}

Index Terms: sequential extraction, potassium reserve, magnesium reserve, heavy metals.

\section{NTRODUÇÃO}

Os depósitos de sedimentos do Grupo Barreiras ocupam a faixa litorânea e parte da sublitorânea de quasetoda a Costa Oriental eSetentrional do Brasil, desde o Rio de J aneiro, estendendo-se para norte, até à Bacia Amazônica (Mabesoone et al., 1972; Bigarella, 1975). Esses sedimentos são de origem fluvial e sua fonte é atribuída ao desmonte de grandes áreas continentais soerguidas (Petri \& Fúlfaro, 1983). A sedimentação ocorreu no Terciário, prolongando-se até o Quaternário. No estado do Espírito Santo, os Sedimentos do Terciário formam os Tabuleiros Costeiros, distribuídos ao longo do litoral, em discordância sobre as rochas do PréCambriano Indiviso ou sobre intrusivas ácidas.

A carência acentuada de nutrientes nos sol os dos tabuleiros deve-se ao fato de as áreas fontes dos sedimentos já estarem bastante intemperizadas na ocasião do transporte e deposição nos Tabuleiros Costei ros (Corrêa, 1984). Como resultado, tem-se um material cujas frações areia e silte são constituídas basicamente de quartzo, com apenas traços de mica (EMBRAPA, 1996; Melo 1998; Duarte et al., 2000). A caulinita é o principal mineral da fração argila dos sedimentos, podendo atingir teores acima de $950 \mathrm{~g} \mathrm{~kg}^{-1}$ em profundidade (Melo et al., 2002a). Outros minerais, tais como: goethita, hematita, anatásio, quartzo e vermiculita com Al-hidróxi entrecamadas, têm sido encontrados nessa fração coloidal (EMBRAPA, 1996). São, portanto, sedimentos estáveis e resistentes ao intemperismo, com pouca reserva de nutrientes (EMBRAPA, 1978; Mattos, 1979).

Contudo, a presença de minerais micáceos na fração argila, além da caulinita, mesmo em pequena quantidade, poderia representar importantereserva de $\mathrm{K}$ e de Mg. Melo et al. (2002a), trabalhando com sedimentos do Grupo Barreiras, em diferentes profundidades, encontrou teores de mica nas frações silte e argila inferiores a $5 \mathrm{~g} \mathrm{~kg}^{-1}$. Ribeiro (1998) e Duarte et al. (2000) também relataram a presença de traços de mica na fração argila de solos desenvolvidos de sedimentos do Grupo Barreiras.

O objetivo deste trabalho foi estudar a distribuição de K, Mg e outros metais em minerais das frações argila, siltee areia no sol o e no sedimento do Grupo Barreiras, no município de Aracruz (ES).

\section{MATERIAL E MÉTODOS}

\section{Amostragem do solo e do sedimento}

As amostras foram coletadas de acordo com a profundidade, em sedimentos do Grupo Barreiras, às margens de um canal adutor de águas, no 
município deAracruz (ES) (19049'13"S 40¹6'24"O). A amostragem foi feita em um único perfil, nas seguintes profundidades (valores após a correção da declividade, $45^{\circ}$, do talude do canal): 0,$7 ; 1,4 ; 2,1$; 2,$8 ; 3,5 ; 4,2 ; 4,9 ; 5,6 ; 7,7 ; 10,5$ e $14 \mathrm{~m}$. O solo dolocal de amostragem foi classificado como Argissolo Amarelo (EMBRAPA, 1999), apresentando a seguinte distribuição e profundidade dos horizontes: A $(0-16 \mathrm{~cm}), A B(16-25 \mathrm{~cm}), \operatorname{Bt}(25-125 \mathrm{~cm}), B C(125-$ $167 \mathrm{~cm})$ e $C\left(1,67-14,0 \mathrm{~m}^{+}\right)$. As duas primeiras amostras, 0,7 e1,4 m, foram tomadas nos horizontes Bt e BC, respectivamente, e as demais no horizonte $C$, encontrando-se o ponto mais profundo cerca de $5 \mathrm{~m}$ acima do leito d'água. Durante a amostragem do horizonte $\mathrm{C}$, procurou-se separar as camadas de sedimentos com características distintas, tais como: cor, presença de concreções de Fe etextura. O recente corte do talude do canal, por ocasião da amostragem, limita a ação dos fatores externos sobre os minerais. $\mathrm{Na}$ amostragem, cerca de $50 \mathrm{~cm}$ da partesuperficial, em cada ponto, foi descartada.

\section{Remoção de matéria orgânica, separação das frações para análises químicas e mineralógicas e análise textural}

As amostras do solo e do sedimento foram tratadas com hipoclorito de sódio para remoção de matéria orgânica e com NaOH 0,2 mol L-1 para dispersão das partículas (J ackson, 1979). Nas amostras coletadas em profundidades maiores que 4,9 m, tanto na análise textural (E MBRAPA, 1979), como na separação das frações para análises químicas e mineralógicas, foi adicionada sol ução de hexametafosfato de sódio ( $5 \%$ p/v), para viabilizar a dispersão. A fração areia foi retida em peneira de $0,05 \mathrm{~mm}$ e as frações argila e silte foram separadas por sedimentação, com base na lei de Stokes (Gee \& Bauder, 1986). Para garantir a separação das frações, o silte foi submetido à dispersão ultra-sônica por 10 min. O material resultante foi recolocado em recipiente próprio para limpeza do silte por mais alguns dias, até que a suspensão sobrenadante ficasse totalmente clara, mantendo-se o $\mathrm{pH}$ da suspensão em 10 , pela utilização de $\mathrm{Na}_{2} \mathrm{CO}_{3}(1 \mathrm{~g} / 10 \mathrm{~L}$ de água desionizada).

\section{Análises químicas}

Teores totais de elementos - Para determinar os teores totais de elementos na terra fina e nas frações areia, silte e argila, utilizou-se o método da digestão com HF concentrado (Lim \& J ackson, 1986, modificado por Melo et al., 2002ab), após secagem das amostras em estufa a $105^{\circ} \mathrm{C}$, por $12 \mathrm{~h}$. Os teores de Mg, Ca, Al, Fe, Ti, Mn, Cu, Zn, Ni, Pb, Cr, Sr e Ga foram determinados em espectrômetro de emissão por plasma Perkins Optima 3000. Os teores de K foram determinados isoladamente em espectrofotômetro de emissão de chama. Os teores de Co e $\mathrm{Cd}$ não foram determinados por problemas analíticos.
Extração seqüencial eseletiva dos minerais Para avaliar a contribuição de cada espécie mineral nos teores totais de K e Mg da fração argila, utilizouse o seguinte esquema de extrações sel etivas e seqüenciais (Melo et al., 2002ab): Argila saturada com Na (12 g) $\rightarrow$ Oxalato deAmônio (OA) 0,2 mol L-1 pH 3,0 (McKeague, 1978) (extração de alumínio e óxidos de ferro de baixa cristalinidade) $\rightarrow$ DitionitoCitrato-Bicarbonato (DCB) (Mehra \& J ackson, 1960) (extração de óxidos de ferro mais cristalinos) $\rightarrow$ $\mathrm{NaOH}$ 0,5 mol L-1 fervente (J ackson et al., 1986, modificado por Melo et al., 2002ab) (extração de alumínia, sílica e aluminossilicato de baixa cristalinidade) $\rightarrow \mathrm{NaOH} 5 \mathrm{~mol} \mathrm{~L}^{-1}$ (Norrish \& Taylor, 1961) (extração de caulinita) $\rightarrow \mathrm{NaHSO}_{4}$ (cristais) (J ackson et al., 1986, com adaptações propostas por Melo et al., 2002ab) (extração de mica e demais minerais do tipo 2:1) $\rightarrow$ digestão total com HF concentrado ( $\mathrm{Lim} \&$ J ackson, 1986) (extração de minerais resistentes, como fel dspato equartzo). A pós cada extração, os teores de K, Mg, Al, Fe e Si foram determinados como descrito anteriormente.

Detalhes sobre os métodos utilizados e as modificações e adaptações propostas para as extrações seqüenciais dos minerais da fração argila podem ser obtidos em Mel o et al. (2002ab).

Foram feitas análises de correlações simples (Pearson) entre os teores totais dos elementos nas frações areia, silte e argila e entre os teores obtidos nas extrações seqüenciais na fração argila, utilizando - programa estatístico SAEG, desenvolvido na Universidade Federal de Viçosa.

\section{RESULTADOS E DISCUSSÃO}

\section{Características morfológicas}

Observou-se grande variação de cor entre os horizontes do solo (Quadro 1). Os horizontes B e BC apresentaram matiz 10Y R e o horizonte $C$ col oração mais avermelhada, com matiz $5 Y R$ entre as profundidades de 2,1 e 4,9 m. Em razão da maior proximidade do lençol freático, a partir de 5,6 m, as condições de mai or umi dade favoreceram a redução e segregação do $\mathrm{Fe}$, condicionando uma coloração variegada dos sedimentos. Na maior profundidade, a matriz do sedimento apresentou coloração totalmente branca pela presença quasequeexclusiva de caulinita (cor neutra, $\mathrm{N} 8 /$ ), com pontos rosados (2,5YR 8/4).

\section{Teores totais de elementos}

Os sedimentos do Grupo Barreiras apresentaram limitada reserva de nutrientes, com baixos teores totais de K, Mg e Ca (Quadro 2). Verificou-se aumento apenas nos teores totais deK na terra fina seca em estufa (TFSE) e na fração argila com a 
Quadro 1. Características morfológicas e análise textural do pacote de sedimentos do Grupo Barreiras no município de Aracruz (ES)

\begin{tabular}{|c|c|c|c|c|c|c|c|c|}
\hline \multirow[b]{2}{*}{ Horizonte } & \multirow[b]{2}{*}{ Profundidade } & \multirow[b]{2}{*}{ Cor seco (Munsell) } & \multirow[b]{2}{*}{ Concreção(1) } & \multicolumn{5}{|c|}{ Fração(2) } \\
\hline & & & & Argila & Silte & AF & AG & AT \\
\hline & $\mathrm{m}$ & & & $\ldots$ & - 9 & $\mathrm{~kg}^{-1}$ & - & - \\
\hline Bt & 0,7 & 10YR $7 / 4$ & & 463 & 52 & 133 & 352 & 485 \\
\hline BC & 1,4 & 10YR $8 / 4$ & & 548 & 66 & 107 & 279 & 386 \\
\hline $\mathrm{C} 1$ & 2,1 & $5 Y R 7 / 4$ & Concreções de Fe & 572 & 58 & 91 & 279 & 370 \\
\hline C2 & 2,8 & $5 Y R 7 / 8$ & Concreções de Fe & 551 & 61 & 90 & 298 & 388 \\
\hline C3 & 3,5 & $5 Y R 7 / 6$ & & 494 & 21 & 96 & 390 & 486 \\
\hline C4 & 4,2 & $5 Y R 7 / 6$ & Concreções de Fe & 547 & 33 & 111 & 309 & 420 \\
\hline C5 & 4,9 & $5 Y R \quad 8 / 4$ & Concreções de Fe & 542 & 39 & 129 & 290 & 419 \\
\hline C6 & 5,6 & Variegada $2,5 Y R$ 8/4 e 7,5YR 8/1 & & 574 & 7 & 109 & 310 & 419 \\
\hline C7 & 7,7 & $5 Y R 8 / 4$ & Concreções de Fe & 266 & 23 & 91 & 620 & 711 \\
\hline C8 & 10,5 & Variegada $2,5 Y R$ 8/4 e 7,5YR 8/1 & Concreções de Fe & 527 & 14 & 86 & 373 & 459 \\
\hline C9 & 14,0 & Variegada 2,5YR 8/4 e N 8/ & & 669 & 4 & 58 & 269 & 327 \\
\hline
\end{tabular}

(1) Descrição morfológica segundo Lemos \& Santos (1996). Amostras 3, 6 e 9: concreções de Fe muito freqüente grande dura irregular vermel ho-escura a lilás. Amostras 4, 7 e 10: concreções de Fe freqüente grande dura irregular vermel ho-escura a lilás. ${ }^{(2)} \mathrm{AF}-$ areia fina, AG - areia grossa e AT - areia total.

profundidade de amostragem, atingindo 427,1 e $618,2 \mathrm{~g} \mathrm{~kg}^{-1}$ a $14 \mathrm{~m}$, respectivamente. De maneira geral, a fração silte apresentou os maiores teores totais de $\mathrm{K}$, Ca e $\mathrm{Mg}$. Contudo, considerando a proporção das frações (Quadro 1), verificou-se quea fração argila foi a mais importante reserva de nutrientes do sol o e do sedimento. Por outro lado, os baixos teores totais de nutrientes na fração areia refletiram a pobreza dos sedimentos do Grupo Barreiras em minerais primários facilmente intemperizáveis.

As correlações significativas entre os teores totais de $\mathrm{K}$ e de $\mathrm{Mg}$ nas frações argila, silte e areia ( $r=0,69^{*}, 0,88^{* * *}$ e $0,80^{* *}$, respectivamente) foram atribuídas à presença de partículas de biotita nestas frações, fato confirmado por Melo et al. (2002a). Os teores de $K$ na fração argila foram menores nas camadas de acúmulo de Fe e de $\mathrm{Mn}\left(r=-0,72^{*}\right.$ e $-0,90^{* * *}$, respectivamente). Por outro lado, na fração areia, a correlação entre $\mathrm{K}$ e Fe e a entre $\mathrm{Mg}$ e Fe foram positivas e significativas $\left(r=0,88^{* * *}\right.$ e $0,84^{* * *}$, respectivamente), ou seja, os teores de $\mathrm{K}$ e de $\mathrm{Mg}$ aumentaram nas camadas de maior impureza do quartzo (maior presença de outros minerais). Da mesma forma, verificou-se alta correlação entre Al e $\mathrm{K}$ e entre $\mathrm{Al}$ e $\mathrm{Mg}\left(r=0,98^{* * *}\right.$ e $0,81^{* *}$, respectivamente). Até à profundidade de $14 \mathrm{~m}$, observouse a formação de três camadas enriquecidas com concreções de Fe visualizadas em campo e em laboratório por observações visuais e por meio de lupa da fração areia, compreendendo as amostras col etadas nas profundidades de 2,1 e 2,8; 4,2 e 4,9 e 7,7 e 10,5 m (Quadro 1). Contudo, a concentração máxima de concreções foi observada nas profundidades de 2,1, 4,2 e 7,7 m. As concreções apresentaram diâmetro superior a $1 \mathrm{~cm}$ e cor vermelho-escura a lilás com fino recobrimento amarel o. Concreções com feições semelhantes em solos desenvolvidos de sedimentos do Grupo Barreiras foram descritas por Amador (1982) e Duarte et al. (2000).

As variações nos teores totais de $\mathrm{Fe}_{2} \mathrm{O}_{3}$ na TFSE enas frações argila, siltee areia com a profundidade (Quadro 3) confirmaram a presença de camadas enriquecidas em $\mathrm{Fe}$, com maiores teores nas profundidades de 2,1; 4,2 e 7,7 m. Para as três camadas de acúmulo deFe, verificou-se o predomínio de concreções do tamanho silte, onde esta fração apresentou os maiores teores totais de $\mathrm{Fe}_{2} \mathrm{O}_{3}$ para as profundidades de 2,1, 4,2 e 7,7 m (138,0; 221,7 e $257,4 \mathrm{~g} \mathrm{~kg}^{-1}$ de $\mathrm{Fe}_{2} \mathrm{O}_{3}$, respectivamente).

A presença deTi nas frações argila, silte e areia (Quadro 3) foi atribuída, principalmente, à dissol ução de óxidos de Ti (anatásio e rutilo). Os teores totais de Zn foram expressivamente superiores aos de Cu. Para as frações silte e areia, os coeficientes de correlação entre os teores totais de Fe e outros microelementos foram significativos: fração silte $\mathrm{Fe} / \mathrm{Zn}=0,72^{*}, \mathrm{Fe} / \mathrm{Cu}=0,77^{* *}, \mathrm{Fe} / \mathrm{Cr}=0,76^{* *}, \mathrm{Fe} / \mathrm{Ga}=$ $0,77^{* *}$ e Fe/ $\mathrm{Pb}=0,66^{*}$, e fração areia - $\mathrm{Fe} / \mathrm{Cr}=0,98^{* * *}$, $\mathrm{Fe} / \mathrm{Ga}=0,90^{* * *}, \mathrm{Fe} / \mathrm{Pb}=0,65^{*}$ e Fe/Sr $=0,96^{* * *}$.

Vários trabal hos têm demonstrado a presença de microelementos na estrutura dos óxidos deferro, por meio de substituição isomórfica (Resende, 1976; Schellman, 1983; Singh \& Gilkes, 1992b; Melo et al., 2001a). As frações argila e silte apresentaram os mai ores teores totais de metais pesados (Quadro 4). $\mathrm{Na}$ fração areia, verificou-se aumento nos teores totais de metais pesados nas camadas de sedimentos enriquecidas com concreções de Fe (2,1; 4,2 e 7,7 m). 
Quadro 2. Teores totais de K, Mg e Ca na terra fina seca em estufa (TFSE) e nas frações argila, silte e areia

\begin{tabular}{|c|c|c|c|c|c|c|c|c|c|c|c|c|}
\hline \multirow{2}{*}{ Profundidade } & \multicolumn{3}{|c|}{ TFSE } & \multicolumn{3}{|c|}{ Argila } & \multicolumn{3}{|c|}{ Silte } & \multicolumn{3}{|c|}{ Areia } \\
\hline & $\mathbf{K}$ & Mg & $\mathrm{Ca}$ & $\mathbf{K}$ & Mg & $\mathrm{Ca}$ & K & Mg & $\mathrm{Ca}$ & K & Mg & $\mathrm{Ca}$ \\
\hline $\mathrm{m}$ & & & & & & $-\mathrm{mg}$ & $g^{-1}$ & & & & & - \\
\hline 0,7 & 151,9 & 148,5 & 107,1 & 301,9 & 188,6 & 88,4 & 190,7 & 260,9 & 549,6 & 10,0 & 36,2 & 36,3 \\
\hline 1,4 & 156,6 & 163,2 & 52,9 & 267,4 & 150,6 & 120,0 & 158,5 & 250,2 & 401,2 & 12,5 & 47,5 & 56,8 \\
\hline 2,1 & 187,7 & 113,6 & 50,0 & 246,8 & 149,4 & 76,9 & 307,4 & 335,5 & 348,7 & 119,1 & 78,6 & 82,6 \\
\hline 2,8 & 165,1 & 119,3 & 29,5 & 260,0 & 149,7 & 146,5 & 332,8 & 330,5 & 365,9 & 15,0 & 48,0 & 29,9 \\
\hline 3,5 & 148,1 & 113,0 & 37,9 & 300,3 & 141,1 & 95,6 & 441,2 & 417,8 & 336,7 & 11,2 & 28,9 & 19,0 \\
\hline 4,2 & 219,1 & 115,4 & 48,8 & 349,8 & 150,6 & 89,7 & 403,5 & 359,6 & 523,4 & 25,0 & 53,6 & 41,5 \\
\hline 4,9 & 215,0 & 138,8 & 28,1 & 388,3 & 158,0 & 148,1 & 528,5 & 383,2 & 168,5 & 21,6 & 69,9 & 43,8 \\
\hline 5,6 & 249,5 & 138,8 & 27,4 & 488,8 & 182,1 & 131,8 & 455,4 & 460,4 & 459,0 & 15,0 & 71,2 & 37,2 \\
\hline 7,7 & 128,2 & 142,8 & 44,8 & 440,4 & 224,3 & 102,1 & 472,3 & 387,1 & 231,0 & 17,9 & 119,0 & 51,1 \\
\hline 10,5 & 193,4 & 113,8 & 43,6 & 414,6 & 197,0 & 84,3 & 169,9 & 328,6 & 191,4 & 16,7 & 36,8 & 40,8 \\
\hline 14,0 & 427,1 & 141,5 & 47,0 & 618,2 & 201,2 & 106,0 & 358,7 & 358,8 & 231,0 & 12,8 & 37,5 & 26,2 \\
\hline
\end{tabular}

Quadro 3. Teores totais de $\mathrm{Fe}_{2} \mathrm{O}_{3}, \mathrm{TiO}_{2}, \mathrm{Mn}$, $\mathrm{Zn}$ e Cu na terra fina seca em estufa (TFSE) e nas frações argila, silte e areia

\begin{tabular}{|c|c|c|c|c|c|c|c|c|c|c|}
\hline \multirow{2}{*}{ Profundidade } & \multicolumn{5}{|c|}{ TFSE } & \multicolumn{5}{|c|}{ Argila } \\
\hline & $\mathrm{Fe}_{2} \mathrm{O}_{3}$ & $\mathrm{TiO}_{2}$ & Mn & $\mathbf{Z n}$ & $\mathbf{C u}$ & $\mathrm{Fe}_{2} \mathrm{O}_{3}$ & $\mathrm{TiO}_{2}$ & Mn & $\mathbf{Z n}$ & $\mathbf{C u}$ \\
\hline $\mathrm{m}$ & \multicolumn{2}{|c|}{$\longrightarrow \mathrm{g} \mathrm{kg}^{-1}$} & \multicolumn{3}{|c|}{$\mathrm{mg} \mathrm{kg}^{-1}$} & \multicolumn{2}{|c|}{$\mathrm{g} \mathrm{kg}^{-1}$} & \multicolumn{3}{|c|}{$\mathrm{mg} \mathrm{kg}^{-1}$} \\
\hline 0,7 & 32,7 & 15,8 & 67,4 & 109,1 & 2,5 & 53,8 & 24,3 & 62,2 & 55,1 & 19,1 \\
\hline 1,4 & 38,4 & 17,7 & 62,4 & 54,1 & 5,8 & 53,2 & 22,7 & 54,5 & 99,8 & 23,3 \\
\hline 2,1 & 62,1 & 21,4 & 64,7 & 76,1 & 6,0 & 53,0 & 22,7 & 50,9 & 102,9 & 11,3 \\
\hline 2,8 & 34,5 & 18,5 & 60,8 & 38,6 & 2,2 & 45,7 & 24,9 & 47,2 & 73,0 & 13,3 \\
\hline 3,5 & 23,4 & 19,1 & 54,6 & 105,6 & 2,7 & 37,5 & 30,4 & 42,3 & 67,9 & 21,3 \\
\hline 4,2 & 54,5 & 20,4 & 62,3 & 69,3 & 4,3 & 44,4 & 27,3 & 34,2 & 59,7 & 16,9 \\
\hline 4,9 & 43,0 & 25,5 & 101,7 & 106,4 & 3,7 & 39,1 & 28,3 & 29,6 & 70,7 & 19,2 \\
\hline 5,6 & 22,3 & 24,4 & 90,8 & 50,4 & 5,1 & 27,6 & 23,8 & 19,5 & 82,7 & 20,9 \\
\hline 7,7 & 42,8 & 25,3 & 181,2 & 82,8 & 5,2 & 47,4 & 21,8 & 23,9 & 71,1 & 38,8 \\
\hline 10,5 & 37,7 & 15,6 & 45,1 & 37,7 & 7,2 & 45,0 & 21,6 & 16,7 & 112,5 & 17,0 \\
\hline \multirow[t]{2}{*}{14,0} & 24,8 & 14,7 & 22,4 & 76,1 & 7,2 & 32,4 & 17,3 & 8,0 & 60,7 & 14,5 \\
\hline & \multicolumn{5}{|c|}{ Silte } & \multicolumn{5}{|c|}{ Areia } \\
\hline 0,7 & 22,9 & 27,3 & 177,4 & 47,4 & 14,3 & 10,8 & 3,3 & 33,9 & 4,5 & 2,4 \\
\hline 1,4 & 27,7 & 25,1 & 150,2 & 80,9 & 7,6 & 9,5 & 4,9 & 41,7 & 4,9 & 1,6 \\
\hline 2,1 & 138,0 & 29,5 & 154,9 & 96,5 & 10,1 & 60,6 & 8,2 & 65,8 & 13,4 & 2,9 \\
\hline 2,8 & 101,7 & 43,1 & 227,9 & 108,4 & 15,9 & 9,5 & 6,7 & 62,8 & 35,1 & 2,7 \\
\hline 3,5 & 72,5 & 59,9 & 345,8 & 95,0 & 22,5 & 3,5 & 3,6 & 28,4 & 5,3 & 3,0 \\
\hline 4,2 & 221,7 & 47,5 & 318,9 & 130,5 & 21,5 & 33,1 & 7,5 & 59,9 & 4,4 & 1,2 \\
\hline 4,9 & 194,9 & 66,3 & 457,5 & 141,4 & 30,5 & 23,7 & 12,6 & 116,2 & 7,4 & 3,9 \\
\hline 5,6 & 82,3 & 79,4 & 577,8 & 142,0 & 23,8 & 9,6 & 12,7 & 119,0 & 2,6 & 4,4 \\
\hline 7,7 & 257,4 & 48,5 & 391,8 & 114,8 & 65,2 & 27,9 & 20,4 & 211,9 & 8,0 & 3,5 \\
\hline 10,5 & 236,4 & 61,3 & 378,0 & 145,7 & 45,5 & 12,7 & $\begin{array}{r}4,8 \\
4,8\end{array}$ & 37,3 & 6,6 & 3,5 \\
\hline 14,0 & 79,6 & 61,4 & 166,4 & 66,0 & 17,5 & 4,3 & 5,8 & 42,7 & 6,8 & 3,9 \\
\hline
\end{tabular}

\section{Liberação de $K$ e de Mg nas extrações seqüenciais da fração argila}

Os teores de $\mathrm{K}$ e de Mg extraídos pelo oxalato de amônio (OA) das amostras col etadas até à profundidade de 4,2 $\mathrm{m}$ foram similares, apresentando menores valores, em relaçãoàs maiores profundidades (Quadro 5). Por outro lado, os teores de Mg não apresentaram grandes variações ao longo dos sedimentos (Quadro 6). Meloet al. (2002b), estudando solos desenvolvidos de sedimentos do Grupo 
Quadro 4. Teores totais de $\mathrm{Ni}, \mathrm{Pb}, \mathrm{Cr}$, $\mathrm{Sr}$ e $\mathrm{Ga}$ na terra fina seca em estufa (TFSE) no sedimento e nas frações argila, silte e areia

\begin{tabular}{|c|c|c|c|c|c|c|c|c|c|c|}
\hline \multirow{2}{*}{ Profundidade } & \multicolumn{5}{|c|}{ TFSE } & \multicolumn{5}{|c|}{ Argila } \\
\hline & $\mathbf{N i}$ & $\mathbf{P b}$ & $\mathbf{C r}$ & Sr & $\mathbf{G a}$ & $\mathbf{N i}$ & $\mathbf{P b}$ & $\mathrm{Cr}$ & Sr & $\mathbf{G a}$ \\
\hline $\mathrm{m}$ & \multicolumn{10}{|c|}{$\mathrm{mg} \mathrm{kg}^{-1}$} \\
\hline 0,7 & 11,4 & 5,3 & 49,6 & 11,1 & 17,7 & 23,9 & 13,8 & 78,2 & 21,3 & 43,3 \\
\hline 1,4 & 11,9 & 22,3 & 62,6 & 13,1 & 24,8 & 21,7 & 22,2 & 79,5 & 20,7 & 46,1 \\
\hline 2,1 & 11,2 & 19,9 & 80,1 & 14,7 & 22,4 & 19,2 & 25,1 & 81,4 & 21,3 & 44,4 \\
\hline 2,8 & 11,0 & 22,3 & 64,3 & 17,4 & 26,7 & 24,8 & 42,7 & 85,4 & 28,6 & 45,2 \\
\hline 3,5 & 13,1 & 31,8 & 65,2 & 19,9 & 31,0 & 24,2 & 67,0 & 104,6 & 37,6 & 59,8 \\
\hline 4,2 & 14,2 & 34,7 & 85,4 & 21,6 & 29,9 & 29,5 & 60,4 & 98,6 & 36,2 & 58,0 \\
\hline 4,9 & 12,5 & 39,9 & 75,5 & 24,2 & 30,7 & 28,8 & 56,5 & 101,3 & 40,3 & 51,8 \\
\hline 5,6 & 13,4 & 38,4 & 63,4 & 22,0 & 24,7 & 31,4 & 58,3 & 93,9 & 34,4 & 55,1 \\
\hline 7,7 & 5,3 & 40,6 & 47,3 & 45,0 & 11,8 & 30,3 & 115,3 & 89,3 & 37,2 & 51,7 \\
\hline 10,5 & 12,7 & 46,0 & 60,2 & 22,8 & 19,8 & 21,2 & 83,9 & 103,7 & 40,7 & 45,8 \\
\hline \multirow[t]{2}{*}{14,0} & 12,6 & 49,0 & 63,7 & 32,7 & 22,1 & 19,8 & 65,7 & 86,7 & 46,6 & 37,8 \\
\hline & \multicolumn{5}{|c|}{ Silte } & \multicolumn{5}{|c|}{ Areia } \\
\hline 0,7 & 0,7 & 8,3 & 32,7 & 11,8 & 0,0 & 2,0 & 0,0 & 12,7 & 0,6 & 1,0 \\
\hline 1,4 & 7,6 & 8,3 & 43,5 & 12,6 & 8,6 & 3,3 & 9,3 & 13,7 & 0,8 & 0,0 \\
\hline 2,1 & 10,3 & 45,4 & 101,2 & 26,0 & 31,8 & 3,8 & 13,9 & 48,9 & 4,2 & 7,7 \\
\hline 2,8 & 19,2 & 48,2 & 139,9 & 44,1 & 53,1 & 0,4 & 9,1 & 13,1 & 1,0 & 1,6 \\
\hline 3,5 & 16,7 & 44,1 & 131,2 & 50,9 & 44,6 & 0,0 & 0,0 & 5,8 & 0,8 & 0,0 \\
\hline 4,2 & 10,7 & 48,5 & 176,4 & 42,1 & 68,2 & 2,4 & 11,3 & 30,5 & 2,3 & 4,4 \\
\hline 4,9 & 13,6 & 42,7 & 162,0 & 45,6 & 69,9 & 3,9 & 8,4 & 23,8 & 2,0 & 2,0 \\
\hline 5,6 & 9,4 & 50,8 & 117,0 & 46,0 & 42,0 & 2,2 & 11,6 & 12,5 & 1,5 & 2,2 \\
\hline 7,7 & 13,1 & 62,0 & 148,6 & 27,7 & 50,6 & 2,3 & 15,9 & 19,4 & 1,9 & 0,8 \\
\hline 10,5 & 10,4 & 54,8 & 125,0 & 30,6 & 52,7 & 2,1 & 4,0 & 8,8 & 1,6 & 0,0 \\
\hline 14,0 & 9,1 & 58,3 & 107,1 & 48,4 & 24,3 & 3,5 & 2,2 & 5,7 & 1,0 & 0,0 \\
\hline
\end{tabular}

Barreiras e outros materiais, concluíram que a liberação de $\mathrm{K}$ pelo OA era devida à extração de minerais al uminos-silicatados de baixa cristal inidade ( $r$ entre $\mathrm{Al}$ e $\mathrm{K}=0,80^{* * *}$ ). J á a liberação de $\mathrm{Mg}$, de acordo com os mesmos autores, foi atribuída à extração de camada octaédrica de baixa cristalinidade que continha $\mathrm{Fe}$ e $\mathrm{Mg}$ ou à presença de $\mathrm{Mg}$ na estrutura dos óxidos de ferro de baixa cristalinidade, ondeo Mg substitui isomorficamente o Fe ( $r$ entre Fe e $\left.\mathrm{Mg}=0,88^{* * *}\right)$.

Contudo, no presente estudo, não foram verificadas essas associações. As correlações entre $\mathrm{Al}$ e $\mathrm{K}$ e entre $\mathrm{Fe}$ e $\mathrm{Mg}$ foram baixas e nãosignificativas ( $r=0,10$ e 0,36, respectivamente). Outra possibilidade é a dissolução de pequenas partículas de biotita, liberando $\mathrm{K}$ e Mg (J ackson et al., 1986). Os teores de $\mathrm{K}$ e de $\mathrm{Mg}$ extraídos pelo ditionito-citrato-bicarbonato (DCB) foram inferiores aos obtidos pelo OA, mostrando que a associação de formas estruturais destes nutrientes com os óxidos de ferro cristalinos foi muito reduzida.

$\mathrm{Na}$ extração com $\mathrm{NaOH} 5 \mathrm{~mol} \mathrm{~L}^{-1}$, os teores de $\mathrm{K}$ e de M g variaram de 122,7 a 227,8 $\mathrm{mg} \mathrm{kg}^{-1}$ a 111,3 a 229,3 $\mathrm{mg} \mathrm{kg}^{-1}$, respectivamente (Quadros 5 e 6). A presença de $\mathrm{K}$ e de $\mathrm{Mg}$ na caulinita foi atribuída à ocorrência de camadas residuais de mica preservadas no interior da estrutura do mineral (Lee et. al., 1975; Lim et al., 1980; Singh \& Gilkes, 1992a). Segundo Melo et al. (2001b), as menores partículas de caulinita na fração argila apresentaram menor grau decristalinidadeetemperatura dedesidroxilação e maiores teores de $\mathrm{K}$ e de $\mathrm{Mg}$.

Os teores de $K$ na fração argila, extraídos com $\mathrm{NaHSO}_{4}$, aumentaram com a profundidade de amostragem e foram, de maneira geral, muito superiores aos de Mg (Quadros 5 e 6). Pequena quantidade de feldspato já seria suficiente para fornecer o K obtido com a extração com HF, considerando uma concentração média de $140 \mathrm{~g} \mathrm{~kg}^{-1}$ deK nos feldspatos potássicos (J ackson, 1979). Melo et al. (2002ab), estudando sol os altamente intemperizados de diferentes regiões do Brasil, por meio de microscopia el etrônica de transmissão e de varredura e microssonda de dispersão de raios-X, identificaram eanalisaram quimicamente partículas de fel dspato presentes nas frações argila e silte de várias classes de Latossolos. Outra possibilidadeéa extraçãopeloHF departículas debiotita queresistiram ao tratamento com $\mathrm{NaHSO}_{4}$, liberando $\mathrm{K}$ e Mg.

A contribuição dos minerais extraídos nos diferentes tratamentos seqüenciais nos teores totais de $\mathrm{K}$ e de $\mathrm{Mg}$ (números entre parênteses nos 
Quadro 5. Teores de K total e obtidos nas extrações seqüenciais na fração argila(1)

\begin{tabular}{|c|c|c|c|c|c|c|c|}
\hline Profundidade & K-total & AO & DCB & NaOH 0,5 & NaOH 5 & $\mathrm{NaHSO}_{4}$ & HF \\
\hline $\mathrm{m}$ & \multicolumn{7}{|c|}{$-\mathrm{mg} \mathrm{kg}^{-1}$} \\
\hline 0,7 & 301,9 & 9,7 (33) & $4,7(16)$ & $21,8(67)$ & $195,6(538)$ & $2.116,2(320)$ & $722,6(27)$ \\
\hline 1,4 & 267,4 & $12,7(47)$ & $3,6(13)$ & 19,9 (67) & $200,0(580)$ & $1.856,1(262)$ & $926,4(31)$ \\
\hline 2,1 & 246,8 & $11,3(46)$ & $3,6(14)$ & $26,1(96)$ & $163,6(524)$ & $2.094,9(278)$ & $1.214,5(43)$ \\
\hline 2,8 & 260,0 & $11,4(44)$ & $3,3(12)$ & $18,4(65)$ & $127,5(387)$ & $3.051,8(450)$ & $1.435,6(42)$ \\
\hline 3,5 & 300,3 & 14,0 (47) & $0,6(02)$ & $17,0(54)$ & 122,7 (334) & $3.215,1$ (513) & $1.424,1(51)$ \\
\hline 4,2 & 349,8 & $13,9(40)$ & $1,6(05)$ & $15,0(40)$ & $151,6(349)$ & $4.301,5(510)$ & $2.001,6(57)$ \\
\hline 4,9 & 388,3 & 19,1 (49) & $6,1(16)$ & $12,3(30)$ & $139,3(302)$ & $5.121,7(562)$ & $1.609,0(41)$ \\
\hline 5,6 & 488,8 & $38,3(78)$ & $4,1(8)$ & $16,4(33)$ & 182,2 (319) & $6.329,2(533)$ & $1.426,0(29)$ \\
\hline 7,7 & 440,4 & $39,0(89)$ & $4,1(9)$ & $15,0(32)$ & $188,7(368)$ & $5.974,3(471)$ & $1.396,8(31)$ \\
\hline 10,5 & 414,6 & $24,3(59)$ & $3,2(8)$ & $10,3(24)$ & 181,9 (375) & $5.542,5(512)$ & $1.080,4(23)$ \\
\hline 14,0 & 618,2 & $21,8(35)$ & $1,2(2)$ & 11,0 (18) & $227,8(324)$ & $5.702,0(571)$ & $1.568,4(51)$ \\
\hline
\end{tabular}

(1) Os valores entre parênteses indicam a contribuição $\left(\mathrm{g} \mathrm{kg}^{-1}\right)$ dos minerais extraidos nos tratamentos seqüenciais em relação ao $\mathrm{K}$ total da fração argila. Extrações seqüenciais: oxalato de amônio (OA), ditionito-citrato-bicarbonato (DCB), $\mathrm{NaOH} 0,5$ mol $\mathrm{L}^{-1}$, $\mathrm{NaOH} 5 \mathrm{~mol} \mathrm{~L}^{-1}, \mathrm{NaHSO}_{4}$ e HF.

Quadro 6. Teores de Mg total e obtidos nas extrações seqüenciais na fração argila(1)

\begin{tabular}{|c|c|c|c|c|c|c|c|}
\hline Profundidade & Mg-total & OA & DCB & NaOH 0,5 & NaOH 5 & $\mathrm{NaHSO}_{4}$ & HF \\
\hline \multicolumn{8}{|l|}{$\mathrm{m}$} \\
\hline 0,7 & 188,6 & $11,9(50)$ & $3,8(15)$ & $0,3(1)$ & $200,3(682)$ & $1.283,1(193)$ & $1.552,4(59)$ \\
\hline 1,4 & 150,6 & $17,2(101)$ & $3,9(22)$ & $0,3(1)$ & $128,1(584)$ & $958,7(203)$ & $1.737,6(88)$ \\
\hline 2,1 & 149,4 & $22,0(139)$ & $4,5(28)$ & $0,0(0)$ & $111,3(558)$ & $1.149,1(225)$ & $967,5(49)$ \\
\hline 2,8 & 149,7 & $12,9(84)$ & $2,6(17)$ & $1,5(9)$ & $115,2(599)$ & 791,1 (197) & $1.928,2$ (95) \\
\hline 3,5 & 141,1 & $15,4(96)$ & $1,6(10)$ & $0,0(0)$ & $119,0(614)$ & 664,9 (199) & $1.206,1(81)$ \\
\hline 4,2 & 150,6 & $14,3(91)$ & $2,0(12)$ & $0,0(0)$ & 126,9 (655) & 695,7 (183) & 930,9 (59) \\
\hline 4,9 & 158,0 & $15,0(93)$ & $1,6(09)$ & $0,0(0)$ & $126,5(659)$ & $712,1(187)$ & $833,9(51)$ \\
\hline 5,6 & 182,1 & $19,7(121)$ & $0,7(04)$ & $0,1(0)$ & $122,3(641)$ & 782,5 (197) & $596,5(36)$ \\
\hline 7,7 & 224,3 & $14,8(85)$ & $2,0(12)$ & $0,0(0)$ & $137,5(668)$ & $885,0(176)$ & $1.042,4(59)$ \\
\hline 10,5 & 197,0 & $8,1(41)$ & $1,9(9)$ & $0,0(0)$ & $173,2(747)$ & $728,4(139)$ & $1.444,0(63)$ \\
\hline 14,0 & 201,2 & $16,2(56)$ & $1,8(6)$ & $0,2(1)$ & $229,3(701)$ & 842,1 (179) & $845,0(58)$ \\
\hline
\end{tabular}

(1) Os valores entre parênteses indicam a contribuição dos minerais $\left(\mathrm{g} \mathrm{kg}^{-1}\right)$ extraídos nos tratamentos seqüenciais em relação ao

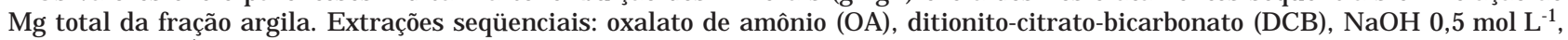
$\mathrm{NaOH} 5 \mathrm{~mol} \mathrm{~L}^{-1}, \mathrm{NaHSO}_{4}$ e HF.

quadros 5 e 6) foram calculados, considerando a quantidade de material removido e os teores de $\mathrm{K}$ e de Mg obtidos em cada extração seqüencial. Maiores detalhes dos cálculos podem ser obtidos em Melo (1998).

Verificou-se limitada associação entre formas estruturais de $\mathrm{K}$ com alumínio e óxidos de ferro de baixa cristalinidade (OA) e óxidos de ferro mais cristalinos (DCB). A participação dos minerais extraídos pelo OA e pelo DCB nos teores totais de K atingiram valores máximos de 89 e $16 \mathrm{~g} \mathrm{~kg}^{-1}$, respectivamente (Quadro 5). Mesmo com baixos teores de K obtidos com NaOH 5 mol L-1, a caulinita contribuiu, nas camadas mais superficiais, com mais de $500 \mathrm{~g} \mathrm{~kg}^{-1}$ do $\mathrm{K}$ total. Essa grande importância na reserva de $K$ foi atribuída à abundância de caulinita na fração argila do solo e do sedimento. J á nos pontos mais profundos de amostragem, os teores de $\mathrm{K}$ e a contribuição da mica (extração com $\mathrm{NaHSO}_{4}$ ) no $\mathrm{K}$ total foram mais expressivos, atingindo $571 \mathrm{~g} \mathrm{~kg}^{-1}$, a $14 \mathrm{~m}$ (Quadro 5). Esse aumento noteor de mica com a profundidade, deveuse, principalmente, ao maior acúmulo de Si, K e outros íons em solução, por limitações de drenagem, tornando o ambiente menos favorável ao intemperismo dos minerais micáceos, comparado com as camadas mais superficiais. U ma evidência deste acúmulo de íons nas maiores profundidades foi a necessidade de adição de solução de hexametafosfato de sódio $(5 \% \mathrm{p} / \mathrm{v})$ a partir da profundidade de 4,9 m, para viabilizar a dispersão das frações argila e silte. Apesar dos altos teores de 
$\mathrm{K}$, a pequena quantidade de material removido pelo HF (Melo et al., 2002a) resultou em uma baixa contribuição dos minerais extraídos pel o tratamento nos teores totais de K (Quadro 5).

Em geral, a participação dos minerais extraídos nos diferentes tratamentos seqüenciais no $\mathrm{Mg}$ total da fração argila foi similar ao observado para o K (Quadros 5 e 6). Os maiores teores de K em relação aos de $\mathrm{Mg}$ obtidos na extração com $\mathrm{NaHSO}_{4}$ foram atribuídos ao predomínio de muscovita, uma vez que essa mica é fonte apenas de $\mathrm{K}$. Como resultado, verificou-se menor partici pação da mica (biotita) no Mg total. O predomínio de muscovita em relação à biotita na fração argila é explicado pelo avançado estádio de intemperismo e maturidade dos sedimentos do Grupo Barreiras. Grande parte do Mg estrutural está na caulinita, chegando a $747 \mathrm{~g} \mathrm{~kg}^{-1}$, a $10,5 \mathrm{~m}$ de profundidade.

\section{CONCLUSÕES}

1. Apesar da pobreza dos sedimentos do Grupo Barreiras em Fe, observaram-se variações nos teores do elemento com a profundidade. Dada a presença decamadas enriquedidas com concreções ferruginosas (principal mente dotamanhosilte), ao longo do pacote de sedimentos, obtiveram-se os maiores teores totais de $\mathrm{Fe}_{2} \mathrm{O}_{3}$, nas profundidades de 2,1; 4,2 e 7,7 m.

2. As frações areia, silte e argila do solo e do sedimento apresentaram limitada reserva de $\mathrm{K}, \mathrm{Mg}$ e Ca. Verificou-se aumento apenas nos teores totais de $\mathrm{K}$ na fração argila com a profundidade de amostragem, atingindo $618,2 \mathrm{mg} \mathrm{kg}^{-1}$ a $14 \mathrm{~m}$.

3. A reserva de $\mathrm{K}$ e de $\mathrm{Mg}$ na fração argila mostrou-se, em grande parte, associada aos minerais mi cáceos e à caulinita. Por outrolado, a contribuição dos óxidos de Fe e de Al e dos aluminossilicatos de baixa cristalinidadee dos óxidos deFemais cristalinos, nos teores totais de $\mathrm{K}$ e de $\mathrm{Mg}$, foi inexpressiva.

\section{LITE RATURA CITADA}

AMADOR, E.S. O Barreiras pleistocênio no Estado do Espírito Santo e seu relacionamento com depósitos de minerais pesados. In: CONGRESSO BRASILEIRO DE GEOLOGIA, 32., Salvador, 1982. Anais. Salvador, Sociedade Brasileira de Geologia, 1982. v.4, p.1462-1472.

BIGARELA, J J. The Barreiras Group in Northeastern Brazil. An. Acad. Bras. Ci., 47:365-393, 1975.

CORRÊA, G.F. Modelo de evolução da mineralogia da fração argila de solos do Planalto de Viçosa, MG. Viçosa, Universidade federal de Viçosa, 1984. 87p. (Tese de Mestrado)
DUARTE, M.N.; CURI, N.; PÉREZ, D.V.; KÄMPF, N. \& CLAESSEN, M.E.C. Mineralogia, química e micromorfologia de sol os de uma microbacia nos Tabuleiros Costeiros do Espírito Santo. Pesq. Agropec. Bras., 35:12371250, 2000.

EMPRESA BRASILEIRA DE PESQUISA AGROPECUÁRIA EMBRAPA. Serviço Nacional de Levantamento e Conservação de Solo. Levantamento de reconhecimento dos solos do Estado do Espírito Santo. Rio de J aneiro, 1978. 461p. (Boletim Técnico, 45)

EMPRESA BRASILEIRA DE PESQUISA AGROPECUÁRIA EMBRAPA. Serviço Nacional de Levantamento e Conservação de Solo. Manual de métodos de análise de sol o. Rio de janeiro, 1979. não paginado.

EMPRESA BRASILEIRA DE PESQUISA AGROPECUÁRIA EMBRAPA. Reunião técnica sobre solos coesos dos Tabuleiros Costeiros. Cruz das Almas, 1996. Anais, Cruz das Almas, 1996. 80p.

EMPRESA BRASILEIRA DE PESQUISA AGROPECUÁRIA EMBRAPA. Centro Nacional de Pesquisas de Solos. Rio de J aneiro. Sistema Brasileiro de classificação de solos. Brasília, Serviço de Produção de Informação - SPI, 1999. $412 \mathrm{p}$.

GEE, G.W. \& BAUDER, J.W. Particle-size analysis. In: KLUTE, A., ed. Methods of soil analysis. Parte 1: Physical and mineralogical methods. Madison, Soil Science Society of America, 1986. p.383-412.

JACKSON, M.L. Soil chemical analysis - advanced course. Madison, Prentice-Hall, 1979. 895p.

JACKSON, M.L.; LIM, C.H. \& ZELAZNY, L.W. Oxides, hydroxides, and aluminosilicates. In: KLUTE, A., ed. Methods of soil analysis. Parte 1: Physical and mineralogical methods. Madison, American Society of Agronomy, 1986. p.101-150.

LEE, S.Y.; J ACKSON, M.L. \& BROWN, J .L. Micaceous oclusions in kaol inite observed by ultramicrotomy and high resolution electron microscopy. Clays Clay Miner., 23:125-129, 1975.

LEMOS, R.C. \& SANTOS, R.D. Manual de descrição e coleta de solos no campo. 3. ed. Campinas, Sociedade Brasileira de Ciência do Solo, 1996. 85p.

LIM, C.H.; J ACKSON, M.L.; KOONS, R.D. \& HELMKE, P.A. Kaolins: sources of differences in cation-exchange capacities and cesium retention. Clays Clay Miner., 28:223-229, 1980.

LIM, C.H. \& J ACKSON, M.L. Dissolution for total elemental analysis. In: PAGE, A.L., ed. Methods of soil analysis. Parte 2: Chemical and microbiological properties. Madison, American Society of Agronomy, 1986. p.1-12.

MABESOONE, J.M.; CAMPOS E SILVA, A. \& BEURLEN, K. Estratigrafia e origem do Grupo Barreiras em Pernambuco, Paraíba e Rio grande do Norte. R. Bras. Geoc., 2:173-188, 1972.

MATTOS, C.M. Propriedades físicas de solos cauliníticos coesos do município de Aracruz, ES. Piracicaba, Escola Superior de Agricultura Luiz de Queiroz, 1979. 73p. (Tese de Mestrado)

McKEAGUE, J.A. Manual on soil sampling and methods of analysis. Ottawa, Canadian Society of Soil Sicience, 1978. $212 \mathrm{p}$. 
MEHRA, O.P. \& J ACKSON, M.L. I ron oxide removal from soils and clay by a dithionite-citrate system bulfered with sodium bicarbonate. Clays Clay Miner., 7:317-327, 1960.

MELO, V.F. Potássio e magnéssio em minerais de solos e relação entre propriedades da caulinita com formas não trocáveis destes nutrientes. Viçosa, Universidade Federal de Viçosa, 1998. 205p. (Tese de Doutorado)

MELO, V.F.; FONTES, M.P.F.; NOVAIS, R.F.; SINGH, B. \& SCHAEFER, C.E.G.R. Características dos óxidos de ferroe de alumínio de diferentes classes de solos. R. Bras. Ci. Solo, 25:19-32, 2001a.

MELO, V.F.; SINGH, B.; SCHAEFER, C.E.G.R.; NOVAIS, R.F. \& FONTES, M.P.F. Chemical and mineralogical properties of kaol initerich Brazilian soils. Soil Sci. Soc. Am. J ., 65:1324$1333,2001 b$.

MELO, V.F.; NOVAIS, R.F.; SCHAEFER, C.E.G.R.; FONTES, M.P.F. \& SINGH, B. Mineralogia das frações areia, silte e argila de sedimentos do Grupo Barreiras no Município de Aracruz, Estado do Espírito Santo. R. Bras. Ci. Solo, 26:29-41, 2002a.

MELO, V.F.; SCHAEFER, C.E.G.R.; NOVAIS, R.F.; SINGH, B. \& FONTES, M.P.F. Potassium and magnesium reserve in clay minerals of some Brazilian soils, as indicated by sequential extraction procedure. Comm. Soil Sci. Plant An., 2002b. (in publication)
NORRISH, K. \& TAYLOR, M. The isomorphous replacement of iron by aluminium in soil goethites. J. Soil Sci., 12:294-306, 1961.

PETRI, S. \& FÚLFARO, V. Geologia do Brasil. São Paulo, Universidade de São Paulo, 1983. 631p.

RESENDE, M. Mineralogy, chemistry, morphology and geomorphology of some soils of Central Plateau of Brazil. West Lafayette, Purdue University, 1976. 237p. (Tese de Doutorado)

RIBEIRO, L.P. Os Latossolos Amarelos do recôncavo Baiano: Gênese, evolução e degradação. Salvador, Seplantec-CADCT, 1998. 99p.

SCHELLMAN, W. Geochemical principles of lateritic nickel ore formation. In: Lateritisation Processes. In: INTERNATIONAL SEMINAR ON LATERITISATION PROCESSES, 2., São Paulo, 1983. Anais. São Paulo, Instituto Astronômico e Geofísico, Universidade de São Paulo, 1983. p.119-135.

SINGH, B. \& GILKES, R.J . Properties of soil kaolinites from south-westen Australia. J. Soil Sci., 43:645-667, 1992a.

SINGH, B. \& GILKES, R.J . Properties and distribution of iron oxides and their asociation with minor elements in the soils of south-western Australia. J. Soil Sci., 43:77-98, 1992b. 
V.F. MELO et al. 